\title{
The early bird gets the worm: benefits and future directions with early antiretroviral therapy initiation in primary HIV infection
}

\author{
Jun Chen ${ }^{1,2,3}$, Rayoun Ramendra ${ }^{1,2,4}$, Hongzhou Lu ${ }^{3}$ \& Jean-Pierre Routy*,1,2,5 \\ ${ }^{1}$ Chronic Viral IIIness Service, McGill University Health Centre, Montreal, QC H4A 3J1, Canada \\ ${ }^{2}$ Research Institute of the McGill University Health Centre, Montreal, QC H4A 3J1, Canada \\ ${ }^{3}$ Department of Infectious Diseases, Shanghai Public Health Clinical Center, Shanghai 201508, PR China \\ ${ }^{4}$ Department of Microbiology \& Immunology, McGill University, Montreal, QC H4A 3J1, Canada \\ ${ }^{5}$ Division of Hematology, Department of Medicine, McGill University Health Centre, Montreal, QC H4A 3J1, Canada \\ *Author for correspondence: Tel.: +1 514843 1558; Fax: +1 514843 1418; jean-pierre.routy@mcgill.ca
}

\begin{abstract}
Primary HIV infection is defined as the first few weeks after infection where plasma viremia is rapidly increasing. Early diagnosis of primary HIV infection enhances the tendency of behavioral changes in newly infected individuals to prevent secondary HIV transmission. Early antiretroviral therapy (ART) benefits individuals by reducing plasma viral load, gut damage, microbial translocation and subsequent systemic immune activation. Early ART leads to the establishment of low HIV reservoir size that may contribute to HIV eradication research. However, substantial diagnostic and logistical barriers remain as a burden to rapid diagnosis and early treatment initiation. In this review, we critically evaluate the effects of early ART and summarize hurdles that must be addressed to implement rapid treatment initiation for newly infected individuals.
\end{abstract}

First draft submitted: 14 June 2018; Accepted for publication: 15 August 2018; Published online: 24 October 2018

Keywords: early ART • HIV screening • primary HIV infection • serological diagnosis of HIV infection • socioeconomic factors

The natural progression of HIV infection is classified into primary, chronic and late stages. These designations are based on the evolution of plasma viral load (VL), HIV-specific immune activation and degree of immunodeficiency [1]. Primary HIV infection (PHI) is accepted as the first few weeks following infection before the establishment of a plateau in plasma VL [1]. The severity of immune damage during this first phase of infection is crucial to determining subsequent disease progression. Therefore, initiating early antiretroviral therapy (ART) to prevent this damage is of great benefit. Indeed, increased antiviral potency, low pill burden and enhanced tolerance of ART have transformed HIV from a fatal disease to a chronic condition. Recently, major improvements in therapeutic management have made early treatment initiation a universal recommendation.

The ramifications of ART initiation during PHI are not well understood as it is difficult to recruit patients during this short and transient state. Therapeutic guidelines from the US Department of Health and Human Services as well as the European AIDS Clinical Society recommend universal treatment for PHI as of 2015 [2,3]. Identifying hurdles to early ART initiation will contribute to the success of the Joint United Nations Program on HIV/AIDS (UNAIDS) 90-90-90 targets stating that 90\% of HIV-infected individuals know their status, 90\% initiate ART and $90 \%$ achieve viral suppression by the year 2020 [4]. Herein, we critically evaluate the effects of early ART initiation on patient and public health while identifying obstacles that must be addressed to achieve early ART initiation for newly infected individuals.

Future Medicine 


\section{Benefits of early ART initiated in PHI}

Early ART initiation slows disease progression \& reduces size of the viral reservoir

Clinically, individuals during PHI present with flu-like syndrome. Increased severity and duration of such symptoms are associated with higher peak viremia and faster disease progression in the absence of treatment [5]. Inhibition of HIV replication by early ART has been shown to attenuate the severity of symptoms.

The persistence of HIV viral reservoir prevents the eradication of HIV even when ART is initiated 10-day postinfection [6. Importantly, ART initiated during PHI was found to increase the first decay slope of reservoir size as assessed by cell-associated HIV DNA [7]. Early ART initiation may contribute to HIV eradication research by maintaining small or undetectable viral reservoirs with low level of immune activation $[7,8]$.

\section{Early ART initiation partially restores HIV-associated gut-associated lymphoid tissue damage}

HIV replication during PHI occurs preferentially in the gut-associated lymphoid tissue, this results in a rapid depletion of CD4 T cells and alterations to the $\alpha 4 \beta 7^{+} \mathrm{CD} 4 \mathrm{~T}$ cells that play a key protective role in the mucosa $[9,10]$. Subsequently, this leads to epithelial gut damage, microbial dysbiosis and subsequent translocation of gut flora into systemic circulation [11,12]. Epithelial gut dysfunction and microbial translocation are associated with disease progression and represent a major contributor to HIV-associated systemic immune activation [13,14]. Early ART only partially restores GI tract damage while reducing systemic immune activation [15].

\section{Impact of early ART initiation on non-AIDS events, morbidity \& mortality}

During HIV infection, the circulating $\mathrm{CD}^{+}{ }^{+}$T-cell population is characterized by a rapid depletion during PHI, followed by a transient recovery and subsequent progressive decline. Le et al. have shown that initiation of ART within this early spontaneous recovery window greatly improved CD4 T-cell recovery, while a 6-month deferral of ART may compromise such revival [16]. A low CD4/CD8 ratio has been associated with long-term increased risk of non-AIDS events, morbidity and mortality [17]. We and others have previously showed that early rather than prolonged duration of ART contributes to the normalization of CD4/CD8 ratio $[18,19]$. Therefore, early ART during PHI may further help reduce the risk of non-AIDS-related events.

\section{Impact of early ART initiation on immune function}

Initiation of ART during PHI partially protects function of immune cells that play an important role in the control of HIV and prevention of other opportunistic infections. T-follicular helper cells $\left(\mathrm{T}_{\mathrm{FH}}\right)$ and $\mathrm{B}$ cells are crucial in the development of antibodies against a wide variety of infections. $\mathrm{T}_{\mathrm{FH}}$-mediated $\mathrm{B}$-cell responses are significantly altered after PHI in the absence of ART [20,21]. This coincides with increased inflammation and a reduction in memory and effector B-cell functions. Early ART prevents immune dysregulation while preserving $\mathrm{T}_{\mathrm{FH}}$ function and B-cell memory in both the blood and gut $[20,21]$. In addition, it also improved immune-regulation markers, such as kynurenine/tryptophan ratio, which has been associated with Kaposi sarcoma, cardiovascular disease and HIV-associated neurocognitive dysfunction $[10,22]$. The earlier ART is initiated, the earlier these inflammatory markers trend to normal and the greater likelihood that the patient can control the virus and resist opportunistic infections.

\section{Early ART in PHI is essential to reduce secondary transmission}

PHI is associated with a rapid increase of plasma VL that is 100 -fold higher than that during the chronic phase [23]. As a result, those in PHI contribute disproportionally to secondary HIV transmission [24]. Using phylogenetic analyses, individuals with PHI were estimated to be the source of $10-50 \%$ of all transmissions in several epidemiological studies [25]. The role of rapid diagnosis and early ART in preventing HIV transmission has been reviewed extensively [25]. Initiating ART in PHI dramatically decreases plasma VL and potential of transmission independent of high-risk sexual behavior [26-28]. Thus, early ART initiation will reduce secondary transmission and help curb the HIV epidemic.

Taken together, early ART initiation in PHI provides advantages regarding both the patient as well as the public. Despite this overwhelming phenomenon, only $20 \%$ of patients from the Montreal Primary HIV-Infection Study Group had ART initiated within 6 months of their first visit in 2010 [29]. Thankfully, this number has sharply increased to over $90 \%$ since the new 2015 guidelines [29]. However, it is evident that there are still barriers that are impeding the upscaling of early ART initiation in some newly infected individuals. 


\section{Barriers to overcome early ART initiation for all}

\section{Access \& improvements in the laboratory diagnosis of PHI}

While diagnosis of PHI is the prerequisite of early ART initiation, it remains a significant challenge. Symptomatic individuals in PHI may seek healthcare, prompting clinicians to perform a screening test most likely in the context of high-risk behavior. Common symptoms include fever, fatigue, pharyngitis and headache - all of which are not HIV-specific [5]. Different symptom-based methods have been developed to identify suspected persons in PHI [30,31]. Recently, a three-symptom score consisting of fever, myalgia and weight loss of more than $2.5 \mathrm{~kg}$ in the 14 days prior to testing was found to be highly predictive of $\mathrm{PHI}$ in a community-based screening program [31]. These symptombased methods may help when allocating resources in settings that do not routinely screen for PHI. However, asymptomatic individuals, who account for around 22\% of those undergoing PHI are missed [5]. Therefore, risk behavior-based screening should be involved to prevent delayed diagnosis in asymptomatic individuals.

While symptoms and high-risk behavior may prompt clinicians to test for HIV infection, the diagnosis of PHI relies on laboratory testing. Since HIV-specific antibody titers can be negative during the primary phase of infection, the point-of-care (POC) antibody test (third-generation test) often fails to detect HIV infection. The fourth-generation assays, which include detection of the p 24 antigen, provide important value for early identification during the second-week postinfection. Therefore, fourth-generation tests are now recommended to use for HIV screening in USA, Canada and Europe [2,3]. However, fourth-generation assays require noncapillary whole-blood or plasma specimens, making it an unfeasible solution in developing countries. The most reliable method to detect PHI remains HIV nucleic acid testing (NAT). The RV217 study, used semiweekly HIV NAT among high-risk individuals in Thailand and East Africa, to successfully identify individuals with PHI [23]. However, the cost of NAT limits its broad implementation and allows it to only be used among high-risk individuals suspected of PHI with negative or indeterminate HIV antigen/antibody test results. Meanwhile, the technically complexities of NAT impede its application in resource-limited settings. Several strategies including use of third-generation tests followed by NAT as well as combining multiple samples for pooled NAT have been proposed and proven to be cost-effective solutions in such populations [25]. Utilization of POC NAT is promising to overcome the barrier of complex procedure. Currently, several POC NAT devices such as the Alere Q, SAMBA ${ }^{\circledR}$ semiquantitative assay, the Liat ${ }^{\circledR}$ HIV Quant VL assay and the Xpert ${ }^{\circledR}$ HIV-1 Qual assay are under development [25].

Rapid diagnosis of PHI is essential to help individuals initiate ART quickly, while false-negative test results may lead people taking preexposure prophylaxis, which increase the risk of development of drug resistance. In addition, it also aids them in the prevention of further HIV transmissions. For example, individuals with PHI could modify their behaviors to limit further HIV transmission by having safer sex and informing their partners to test and use postexposure prophylaxis if they have recently been exposed to HIV.

To accelerate early ART in PHI, accurate, rapid and cheap methods are still needed to upscale both screening and confirmatory diagnosis. Currently, integration of symptoms, CD4 T-cell count, CD4/CD8 ratio and fourth-generation testing may be helpful, especially in resource-limited settings. Furthermore, targeting high-risk populations by providing frequent HIV testing may help optimize identification of individuals with PHI.

\section{Same-day HIV testing \& initiation of ART}

The attrition rates were as high as 30\% in the time between HIV diagnosis and ART initiation in the 2000s when CD4 T-cell counts were a major guideline for ART initiation [32]. Currently, in the universal treatment era, early ART initiation may still be impeded by the requirement of several visits for HIV screening, laboratory testing and counseling prior to initiating treatment. Reducing these barriers will accelerate access to early ART initiation for all. For example, in Uganda, the presence of a multicomponent intervention targeting healthcare workers prompted $80 \%$ of the eligible individuals to initiate ART within 2 weeks of infection as opposed to only $38 \%$ initiating ART in the control group [33]. One strategy to reduce the time from diagnosis to treatment is to provide same-day ART initiation with HIV testing. The RapIT study conducted in South Africa offered individuals POC CD4 T-cell count, blood test counseling and treatment at their first visit. This increased uptake of ART by $36 \%$ and viral suppression by 26\% 1-year after implementation [34]. Although this strategy was more expensive per individual to initiate treatment, in the long run, it proved to be a more cost-effective means for individuals to achieve viral suppression [35]. In a randomized controlled study conducted in Haiti, investigators determined that individuals who underwent same-day ART initiation had 1.21-times greater ability to retain plasma VL less than 50 copies/ml 1 -year after first visit as compared to control groups. This clearly indicates the benefits of this strategy [36]. 
Recently, research from Lesotho-assessed people who were tested at home and offered same-day ART in regard to their linkage to care and viral suppression [37]. At 12 months, 50.4\% of the participants in the same-day group achieved viral suppression as opposed to $34.3 \%$ of those in the control group [37]. Based on this data, same-day ART initiation has been recommended in the 2017 WHO guidelines to fight the HIV epidemic. This new therapeutic strategy is regarded as one of the most crucial cornerstones in successfully achieving the 90-90-90 targets [4,38].

However, few studies have evaluated these approaches among people in PHI. Girometti et al. reported on 113 individuals in PHI, the majority (77\%) of whom started ART at first medical appointment [39]. None of the individuals discontinued ART by 24 weeks and $99 \%$ of them achieved viral suppression with a median time to documented VL suppression of 74 days [39]. The RAPID study from San Francisco was initially designed to target people during PHI and recent HIV infection ( $<6$ months). Later, the study population was expanded to individuals at any stage of infection [40]. This same-day ART initiation strategy was shown to be feasible and able to shorten the time before viral suppression. Hoenigl et al. reported on $36 \mathrm{PHI}$ individuals who initiated ART at first visit post-HIV diagnosis and had rapid and reliable viral suppression [41]. Another study from Vancouver showed 16 out of 19 individuals with PHI who elected the rapid referral program, which links them to care within a day postdiagnosis [42]. Comparing with this rapid treatment initiation, 14 cases of newly diagnosed chronic HIV infection necessitated an average of 14 days to access to care and treatment [42].

Except structural barriers, newly diagnosed PHI individuals may also experience acute psychological crisis (e.g., fear, anxiety and stigma), which hinder their acceptance of immediate ART initiation [43]. Therefore, to facilitate rapid ART initiation, the whole healthcare system should be patient-centered. Same-day access to HIV specialist should be appointed upon HIV diagnosis. Integrated support with nurses, social workers regarding sexual health and adherence education as well as counseling from a physiologist when needed to cope with the new diagnostic can be provided on the same day. Prepared ART regimens should be offered once laboratory tests are done, which should be modified when the test results are available [40]. Importantly, medical insurance and payment for those without insurance also need to be accelerated. By implementing these procedures, same-day ART initiation at the time of HIV testing in PHI is feasible and can be scaled up to achieve faster viral control. Future efforts should be made to expand this strategy in all PHI individuals, especially those in developing countries.

\section{HIV-infected individual attitudes \& behaviors}

While technological and financial complications represent substantial barriers to early ART initiation, the attitudes of HIV-infected individuals are still the most significant barrier to achieving early ART initiation for all. We have found, as well as others, that a lower CD4 T-cell count is associated with initiation of ART within 6 months of HIV diagnosis [29] This result was consistent with studies surrounding chronic infection in different demographic areas and populations. Hanna et al. showed that lower CD4 T-cell count was linked with shorter time to ART initiation among 10,692 HIV-infected individuals enrolled in North America for the period from 2001 to 2009 [44]. Fatukasi et al. further confirmed this link among 4907 ART-naive individuals enrolled between 2003 and 2012 in the USA [45]. Developing countries, such as South Africa, also associated lower CD4 T-cell count with early ART initiation in 514 HIV-infected individuals enrolled in a universal test and treatment program [46]. Low CD4 T-cell count is likely the result of severe disease progression, making patients feel sicker and prompting them to seek early treatment. However, it is evident that the benefits of early ART outweigh other factors (e.g., stigma, drug toxicities and cost of treatment) in the individuals mind when they are in a relatively good immune state. Therefore, the benefits of early ART should be emphasized to newly diagnosed individuals for direct health benefits to the patient and prevention of HIV transmission.

Socioeconomic status is often considered to be a significant hurdle to early ART initiation in settings where the government or insurance companies do not fully cover the cost of ART. However, we found that even in countries with universal access to care like Canada, having a paid employment was associated with early ART initiation [29]. While having employment may be a surrogate marker for a better understanding of the realities of HIV-infection, it is evident that socioeconomic status plays a significant role in early ART initiation regardless of the cost of treatment. Consistent with our results, Joseph et al. also reported that informal income generation was negatively associated with ART initiation among HIV-positive individuals who use injection drugs in Canada [47]. Similarly, Kesselring et al. found a positive correlation between lower socioeconomic status and late ART initiation in HIV-positive individuals in British Columbia, Canada [48]. In another study from Spain where ART is fully covered by health insurance, Perez-Molina $e t$ al. reported an association between lower socioeconomic status and late ART initiation [49]. In fact, socioeconomic and ART initiation impacts each other mutually. In a Ugandan 
study, health coverage, improved employment and education were reported among HIV-infected adults with early initiation of ART [50].

To accelerate ART initiation, socioeconomic status should be taken into consideration. Social support programs especially financial aids for targeted information and transportation to healthcare centers for individuals with lower socioeconomic status may reduce such barriers to early ART initiation.

\section{Conclusion \& future perspective}

Initiation of treatment during PHI is an undisputed approach to reduce immune damage and tendencies of secondary transmission. However, there are substantial diagnostic, logistic and socioeconomic barriers to implementation and large-scale application of this therapeutic strategy. Future research needs to prioritize the diagnosis of PHI, rapidly and accurately to prompt early ART initiation.

The technology barriers could be tackled in the near future if enough funding is inputted. However, structural barriers, stigma as well as unawareness of the benefit of early ART among PHI individuals may still exist that hamper scale up of early ART. Ideal logistic procedures of early ART need to be proposed in each countries or cities based on the varying PHI prevalence, capacity of healthcare system and cost-effective analysis. Stigma and discrimination will also still prevent healthcare service from reaching PHI individuals. Emphasizing the rights of PHI individuals and integrating social programs may help eradicating stigma. Social programs need to target high-risk populations and provide information on the benefit of frequent HIV testing and early ART initiation. Educate to improve the knowledge of early ART will be able to change the attitude of PHI individuals. To this end, community representatives, healthcare providers and clinicians should work together in programs that integrate HIV screening and treatment.

\section{Executive summary}

\section{Primary HIV infection}

- Primary HIV infection (PHI) is defined as the first few weeks following HIV acquisition. Initiating antiretroviral therapy (ART) during this period will allow for maximum health benefits to the patient, while also reducing the occurrence of secondary HIV transmission.

Benefits of early ART during PHI

- Attenuate gut damage and microbial translocation, thus alleviating HIV-associated inflammation.

- Improve immunological recovery.

- Lower HIV reservoir size.

- Decrease the transmission of HIV by lowering HIV viral load in bodily fluids.

Factors \& barriers linked to early ART in PHI

- Lack of rapid, simple and cheap diagnostic tools.

- Structural barriers that increase duration between HIV testing and ART initiation.

- Accessibility of first-line antiretroviral drugs in developing countries.

- Socioeconomic factors that influence the attitude of HIV-infected individuals.

- Abrogating stigma link to HIV.

\section{Acknowledgements}

The authors acknowledge the contribution of Angie Massicotte, Mario Legault for administrative assistance.

\section{Author's contributions}

J-P Routy designed the review, J Chen drafted the review, R Ramendra and H Lu critically improved the review.

Financial \& competing interest disclosure

This study was supported by the Fonds de recherche du Québec-Santé (FRQ-S): Réseau SIDA/Maladies infectieuses and Thérapie cellulaire; the Canadian Institutes of Health Research (CIHR; grants MOP 103230 and 154051); the Vaccines \& Immunotherapies Core of the CIHR Canadian HIV Trials Network (CTN; grant CTN 257); the Canadian Foundation for AIDS Research (CANFAR; grant 02-512); and the Canadian HIV Cure Enterprise Team Grant (HIG-133050) awarded by the CIHR in partnership with CANFAR. J Chen is supported by CTN postdoctoral fellowship award, J-P Routy is the holder of the Louis Lowenstein Chair in Hematology and Oncology, McGill University. The authors have no other relevant affiliations or financial involvement with any organization or 
entity with a financial interest in or financial conflict with the subject matter or materials discussed in the manuscript apart from those disclosed.

No writing assistance was utilized in the production of this manuscript

\section{Open access}

This work is licensed under the Attribution-NonCommercial-NoDerivatives 4.0 Unported License. To view a copy of this license, visit http://creativecommons.org/licenses/by-nc-nd/4.0/

\section{References}

Papers of special note have been highlighted as: $\bullet$ of interest; $\bullet \bullet$ of considerable interest

1 Cohen MS, Shaw GM, McMichael AJ, Haynes BF. Acute HIV-1 infection. N. Engl. J. Med. 364(20), 1943-1954 (2011).

2 EACS. 2017 European AIDS Clinical Society (EACS) Guidelines for the treatment of adult HIV-positive persons version 9.0. (2017). www.eacsociety.org/files/guidelines_9.0-english.pdf

3 Department of Health and Human Services. Panel on Antiretroviral Guidelines for Adults and Adolescents Guidelines for the use of antiretroviral agents in HIV-1-infected adults and adolescents. (2018). https://aidsinfo.nih.gov/contentfiles/lvguidelines/adultandadolescentgl.pdf

4 Joint United Nations Programme on HIV/AIDS (UNAIDS). Ending AIDS - progress towards the 90-90-90 targets. (2017). www.unaids.org/sites/default/files/media_asset/Global_AIDS_update_2017_en.pdf

5 Crowell TA, Colby DJ, Pinyakorn S et al. Acute retroviral syndrome is associated with high viral burden, CD4 depletion, and immune activation in systemic and tissue compartments. Clin. Infect. Dis. 66(10), 1540-1549 (2018). www.ncbi.nlm.nih.gov/pubmed/29228130

6 Colby DJ, Trautmann L, Pinyakorn S et al. Rapid HIV RNA rebound after antiretroviral treatment interruption in persons durably suppressed in Fiebig I acute HIV infection. Nat. Med. 24, 923-926 (2018).

- Extremely early antiretroviral therapy (ART) in primary HIV infection (PHI) does not eradicate HIV.

7 Laanani M, Ghosn J, Essat A et al. Impact of the timing of initiation of antiretroviral therapy during primary HIV-1 infection on the decay of cell-associated HIV-DNA. Clin. Infect. Dis. 60(11), 1715-1721 (2015).

8 Novelli S, Lecuroux C, Avettand-Fenoel V et al. Long-term therapeutic impact of the timing of antiretroviral therapy in patients diagnosed with primary human immunodeficiency virus type 1 infection. Clin. Infect. Dis. 66(10), 1519-1527 (2018).

9 Planas D, Zhang Y, Monteiro P et al. HIV-1 selectively targets gut-homing CCR6+CD4+ T cells via mTOR-dependent mechanisms. JCI Insight. 2(15), pii: 93230 (2017).

10 Jenabian MA, El-Far M, Vyboh K et al. Immunosuppressive tryptophan catabolism and gut mucosal dysfunction following early HIV infection. J. Infect. Dis. 212(3), 355-366 (2015).

11 McMichael AJ, Borrow P, Tomaras GD, Goonetilleke N, Haynes BF. The immune response during acute HIV-1 infection: clues for vaccine development. Nat. Rev. Immunol. 10(1), 11-23 (2010).

12 Mehraj V, Jenabian MA, Ponte R et al. The plasma levels of soluble ST2 as a marker of gut mucosal damage in early HIV infection. AIDS 30(10), 1617-1627 (2016).

13 Hunt PW, Sinclair E, Rodriguez B et al. Gut epithelial barrier dysfunction and innate immune activation predict mortality in treated HIV infection. J. Infect. Dis. 210(8), 1228-1238 (2014).

14 Krastinova E, Lecuroux C, Leroy C et al. High soluble CD14 levels at primary HIV-1 infection predict more rapid disease progression. J. Infect. Dis. 212(6), 909-913 (2015).

15 Schuetz A, Deleage C, Sereti I et al. Initiation of ART during early acute HIV infection preserves mucosal Th17 function and reverses HIV-related immune activation. PLoS Pathog. 10(12), e1004543 (2014).

16 Le T, Wright EJ, Smith DM et al. Enhanced CD4+ T-cell recovery with earlier HIV-1 antiretroviral therapy. N. Engl. J. Med. 368(3), 218-230 (2013).

17 Buggert M, Frederiksen J, Noyan K et al. Multiparametric bioinformatics distinguish the CD4/CD8 ratio as a suitable laboratory predictor of combined T cell pathogenesis in HIV infection. J. Immunol. 192(5), 2099-2108 (2014).

18 Cao W, Mehraj V, Trottier B et al. Early initiation rather than prolonged duration of antiretroviral therapy in HIV infection contributes to the normalization of CD8 T-cell counts. Clin. Infect. Dis. 62(2), 250-257 (2016).

- $\quad$ Early ART in PHI contributes to CD4/CD8 ratio normalization.

19 Opportunistic Infections Project Team of the Collaboration of Observational HIV Epidemiological Research in Europe in EuroCoord, Young J, Psichogiou M et al. CD4 cell count and the risk of AIDS or death in HIV-infected adults on combination antiretroviral therapy with a suppressed viral load: a longitudinal cohort study from COHERE. PLoS Med. 9(3), e1001194 (2012).

20 Muir R, Metcalf T, Tardif V et al. Altered memory circulating T follicular helper-B cell interaction in early acute HIV infection. PLoS Pathog. 12(7), e1005777 (2016). 
21 Planchais C, Hocqueloux L, Ibanez C et al. Early antiretroviral therapy preserves functional follicular helper T and HIV-specific B cells in the gut mucosa of HIV-1-infected individuals. J. Immunol. 200(10), 3519-3529 (2018).

22 Chen J, Shao J, Cai R et al. Anti-retroviral therapy decreases but does not normalize indoleamine 2,3-dioxygenase activity in HIV-infected patients. PLoS ONE 9(7), e100446 (2014).

23 Robb ML, Eller LA, Kibuuka $\mathrm{H}$ et al. Prospective study of acute HIV-1 infection in adults in East Africa and Thailand. N. Engl. J. Med. 374(22), 2120-2130 (2016).

- The viral-load set point correlates with peak viremia.

24 Hollingsworth TD, Pilcher CD, Hecht FM, Deeks SG, Fraser C. High transmissibility during early HIV infection among men who have sex with men - San Francisco, California. J. Infect. Dis. 211(11), 1757-1760 (2015).

25 Rutstein SE, Ananworanich J, Fidler $\mathrm{S}$ et al. Clinical and public health implications of acute and early HIV detection and treatment: a scoping review. J. Int. AIDS Soc. 20(1), 21579 (2017).

- Review on the clinical and public health perspective of early ART in PHI.

26 Quinn TC, Wawer MJ, Sewankambo N et al. Viral load and heterosexual transmission of human immunodeficiency virus type 1. Rakai Project Study Group. N. Engl. J. Med. 342(13), 921-929 (2000).

27 Ananworanich J, Eller LA, Pinyakorn S et al. Viral kinetics in untreated versus treated acute HIV infection in prospective cohort studies in Thailand. J. Int. AIDS Soc. 20(1), 21652 (2017).

28 Kroon E, Phanuphak N, Shattock AJ et al. Acute HIV infection detection and immediate treatment estimated to reduce transmission by 89\% among men who have sex with men in Bangkok. J. Int. AIDS Soc. 20(1), 21708 (2017).

29 Mehraj V, Cox J, Lebouche B et al. Socio-economic status and time trends associated with early ART initiation following primary HIV infection in Montreal, Canada: 1996 to 2015. J. Int. AIDS Soc. 21(2), doi: 10.1002/jia2.25034 (2018).

-. Socioeconomic status determines the time of ART initiation in PHI.

30 Hoenigl M, Green N, Camacho M et al. Signs or symptoms of acute HIV infection in a cohort undergoing community-based screening. Emerg. Infect. Dis. 22(3), 532-534 (2016).

31 Lin TC, Gianella S, Tenenbaum T, Little SJ, Hoenigl M. A simple symptom score for acute HIV infection in a San Diego community based screening program. Clin. Infect. Dis. 67(1), 105-111 (2018).

- $\quad$ Establishing a 3-symptom-based screening for PHI.

32 Rosen S, Fox MP. Retention in HIV care between testing and treatment in sub-Saharan Africa: a systematic review. PLoS Med. 8(7), e1001056 (2011).

33 Amanyire G, Semitala FC, Namusobya J et al. Effects of a multicomponent intervention to streamline initiation of antiretroviral therapy in Africa: a stepped-wedge cluster-randomised trial. Lancet HIV 3(11), e539-e548 (2016).

34 Rosen S, Maskew M, Fox MP et al. Initiating antiretroviral therapy for HIV at a patient's first clinic visit: the RapIT randomized controlled trial. PLoS Med. 13(5), e1002015 (2016).

35 Long LC, Maskew M, Brennan AT et al. Initiating antiretroviral therapy for HIV at a patient's first clinic visit: a cost-effectiveness analysis of the rapid initiation of treatment randomized controlled trial. AIDS 31(11), 1611-1619 (2017).

36 Koenig SP, Dorvil N, Devieux JG et al. Same-day HIV testing with initiation of antiretroviral therapy versus standard care for persons living with HIV: a randomized unblinded trial. PLoS Med. 14(7), e1002357 (2017).

-• Same-day ART with HIV testing improves retention in care with virologic suppression among patients with early clinical HIV disease.

37 Labhardt ND, Ringera I, Lejone TI $e$ t al. Effect of offering same-day ART vs usual health facility referral during home-based HIV testing on linkage to care and viral suppression among adults with HIV in Lesotho: the CASCADE randomized clinical trial. JAMA 319(11), 1103-1112 (2018).

-• Same-day ART during home-based HIV testing improves linkage to care and viral suppression.

38 WHO. Guidelines for Managing Advanced HIV Disease and Rapid Initiation of Antiretroviral Therapy. WHO, Geneva, Switzerland (2017).

39 Girometti N, Nwokolo N, McOwan A, Whitlock G. Outcomes of acutely HIV-1-infected individuals following rapid antiretroviral therapy initiation. Antivir. Ther. 22(1), 77-80 (2017).

40 Pilcher CD, Ospina-Norvell C, Dasgupta A et al. The effect of same-day observed initiation of antiretroviral therapy on HIV viral load and treatment outcomes in a US Public Health Setting. J. Acquir. Immune Defic. Syndr. 74(1), 44-51 (2017).

41 Hoenigl M, Chaillon A, Moore DJ et al. Rapid HIV viral load suppression in those initiating antiretroviral therapy at first visit after HIV diagnosis. Sci. Rep. 6, 32947 (2016).

42 Thumath M, Sandstra I, Forrest J et al. Implementation of a rapid referral pathway to HIV treatment for gay men and MSM diagnosed with acute HIV-infection in sexual health clinics in British Columbia. 8th IAS Conference on HIV Pathogenesis, Treatment and Prevention. BC, Canada, 19-22 July 2015 (Abstract TUPED782).

43 Christopoulos KA, Massey AD, Lopez AM et al. "Taking a half day at a time:" patient perspectives and the HIV engagement in care continuum. AIDS Patient Care STDS 27(4), 223-230 (2013). 
44 Hanna DB, Buchacz K, Gebo KA et al. Trends and disparities in antiretroviral therapy initiation and virologic suppression among newly treatment-eligible HIV-infected individuals in North America, 2001-2009. Clin. Infect. Dis. 56(8), 1174-1182 (2013).

45 Fatukasi TV, Cole SR, Moore RD et al. Risk factors for delayed antiretroviral therapy initiation among HIV-seropositive patients. PLoS ONE 12(7), e0180843 (2017).

46 Boyer S, Iwuji C, Gosset A et al. Factors associated with antiretroviral treatment initiation amongst HIV-positive individuals linked to care within a universal test and treat programme: early findings of the ANRS 12249 TasP trial in rural South Africa. AIDS Care 28(Suppl. 3), 39-51 (2016).

47 Joseph B, Wood E, Hayashi K et al. Factors associated with initiation of antiretroviral therapy among HIV-positive people who use injection drugs in a Canadian setting. AIDS 30(6), 925-932 (2016).

48 Kesselring S, Parashar S, Kaida A, Cui Z, Oliveira N, Colley G. Socio-economic and clinical factors associated with late initiation of antiretroviral therapy: preliminary results from the ENGAGE Cohort Study. 8th International AIDS Conference (IAS). BC, Canada, 19-22 July 2015 (Abstract WEPED856).

49 Perez-Molina JA, Suarez-Lozano I, Del Arco A et al. Late initiation of HAART among HIV-infected patients in Spain is frequent and related to a higher rate of virological failure but not to immigrant status. HIV Clin. Trials 12(1), 1-8 (2011).

50 Thirumurthy H, Chamie G, Jain V et al. Improved employment and education outcomes in households of HIV-infected adults with high CD4 cell counts: evidence from a community health campaign in Uganda. AIDS 27(4), 627-634 (2013). 